\title{
Cocoa intake attenuates renal injury in Zucker Diabetic Fatty rats by improving glucose homeostasis
}

David Álvarez-Cilleros ${ }^{1}$, Elvira López-Oliva ${ }^{2}$, Luis Goya ${ }^{1}$, María Ángeles Martín ${ }^{1,3}$, and Sonia Ramos ${ }^{1, *}$

${ }^{1}$ Department of Metabolism and Nutrition

Institute of Food Science and Technology and Nutrition (ICTAN)

Consejo Superior de Investigaciones Científicas (CSIC)

José Antonio Novais 10; Ciudad Universitaria, 28040 Madrid. Spain

Phone: +34.91.544.56.07

Fax: +34.91.549.36.27

${ }^{2}$ Sección Departamental de Fisiología. Facultad de Farmacia, Universidad Complutense de Madrid (UCM)

${ }^{3}$ Centro de Investigación Biomédica en Red de Diabetes y Enfermedades Metabólicas Asociadas (CIBERDEM), ISCIII, Spain.

* Corresponding author: e-mail: s.ramos@ictan.csic.es

Abbreviations: AUC, area under curve; eGFR, estimated glomerular filtration rate; GLUT, glucose transporter; G-6-Pase, glucose-6-phosphatase; GS, glycogen synthase; GSK-3, glycogen synthase kinase-3; GTT, glucose tolerance test; Hb1 Ac, hemoglobin glycosylated; H\&E, hematoxylin and eosin; HOMA-IR, homeostasis model assessment of insulin resistance; HOMA-IS, homeostasis model assessment of insulin sensitivity; IR, insulin receptor; PAS, periodic-acid-Schiff; PEPCK, phosphoenolpyruvate carboxykinase; PFA, paraformaldehyde; SGLT-2, sodium-glucose co-transporter-2; ZDF, Zucker diabetic rat; ZL, Zucker Lean. 


\section{Abstract}

Glucotoxicity (high levels of glucose) is a major factor in the pathogenesis of diabetic kidney disease. Cocoa has anti-diabetic effects by lowering glucose levels. However, whether cocoa exerts beneficial effects on the renal cortex glucose homeostasis and the molecular mechanisms responsible for this possible protective activity remain largely unknown. Thus, the potential anti-diabetic properties of cocoa on insulin signalling, glucose transporters and gluconeogenic enzymes were evaluated in the renal cortex of Zucker Diabetic fatty (ZDF) rats. Male ZDF rats were fed a control or cocoa-rich diet (10\%), and Zucker Lean animals received the control diet. ZDF rats supplemented with cocoa (ZDF-Co) showed decreased body weight gain, glucose and insulin levels, improved glucose tolerance, insulin resistance and structural alterations in renal cortex. Moreover, cocoa-rich diet ameliorated insulin resistance by reverting decreased tyrosine-phosphorylated-insulin receptor levels and by preventing the inactivation of glycogen synthase kinase-3/glycogen synthase pathway (GSK-3/GS) in the renal cortex of ZDF-Co rats. Cocoa antihyperglycaemic effect also appeared to be mediated through the diminution of phosphoenolpyruvate-carboxykinase (PEPCK), glucose-6phosphatase (G-6-Pase), sodium-glucose-co-transporter-2 (SGLT-2), and glucosetransporter-2 (GLUT-2) levels in ZDF-Co rat's renal cortex. These findings demonstrate that cocoa alleviates renal injury by contributing to maintain the glucose homeostasis in type 2 diabetic ZDF rats.

Keywords: Cocoa, Glucose homeostasis, Glucose tolerance, Insulin resistance, Renal cortex structural alterations, Type 2 diabetic ZDF rats. 


\section{Highlights}

- Cocoa intake improves glucose tolerance and alleviates insulin resistance in ZDF rats.

- Cocoa-enriched diet protects against structural diabetes-induced changes in the renal cortex of ZDF rats.

- Cocoa intake averts the insulin signalling blockage in the renal cortex of ZDF rats.

- Cocoa improves the enhanced levels of gluconeogenic enzymes in the renal cortex of ZDF rats.

- Cocoa-enriched diet avoids the increased content of glucose transporters in the renal cortex of ZDF rats. 


\section{Introduction}

Diabetic kidney disease is one of the main causes of death in diabetic patients, and affects 15-25\% of them (Persson, F. and Rossing, P., 2018, Winocour, P.H., 2018). Clinically, diabetic kidney disease is characterized by a progressive renal damage accompanied by increasing albuminuria and subsequent impaired renal function, as altered glomerular filtration rate (GFR), among other modifications. Morphologically, different changes occur, such as mesangial expansion and thickening of the glomerular basement membrane, later followed by glomerulosclerosis (Forbes, J.M. and Cooper, M.E., 2013, Persson, F. and Rossing, P., 2018, Thomas, M.C. et al., 2015). Nevertheless, the proximal tubules, which constitute more than $90 \%$ of the cortical mass of the kidney, are especially sensitive to the injury and suffer the greatest changes in growth during diabetes, become atrophic and their basement membranes thickened (Forbes, J.M. and Cooper, M.E., 2013). Importantly, glucotoxicity, i.e. hyperglycaemia or sustained high levels of glucose, is recognized as a main factor in these pathological alterations (Forbes, J.M. and Cooper, M.E., 2013, Persson, F. and Rossing, P., 2018, Thomas, M.C. et al., 2015).

Kidneys play a relevant role in the maintenance of glucose homeostasis (Forbes, J.M. and Cooper, M.E., 2013). During diabetes gluconeogenesis and glucose uptake are increased in the renal cortex, and also the insulin signalling pathway seems to be altered, contributing all these pathological changes to sustain the high levels of glucose. (Artunc, F. et al., 2016, Mitrakou, A., 2011, Rahmoune, H. et al., 2005). Consequently, the achievement of an optimal glycaemic control constitutes the most effective way to prevent or reduce the risk for diabetic complications, including the diabetic kidney disease. 
Cocoa and its derived products are widely consumed in Europe and the United States (Vinson, J.A. et al., 2006), and have shown beneficial effects against diabetes in humans (Costa, C. et al., 2017, Grassi, D. et al., 2008, Kim, Y. et al., 2016, Martín, M.A. et al., 2016, Martín, M.A. et al., 2017). Accordingly, cocoa and its flavanols have demonstrated anti-diabetic effects related to their ability to improve the insulin sensitivity (Álvarez-Cilleros, A. et al., 2018, Cordero-Herrera, I. et al., 2015, CorderoHerrera, I. et al., 2014, Grassi, D. et al., 2008, Zhang, Z. et al., 2013), to contribute to the glucose homeostasis, which was associated to decreased blood glucose levels (Cordero-Herrera, I. et al., 2015, Grassi, D. et al., 2008, Jalil, A.-M.-M. et al., 2008, Muthenna, P. et al., 2014, Ruzaidi, A. et al., 2005), and to protect renal functionality, and structure (Igarashi, K. et al., 2007, Muthenna, P. et al., 2014, Papadimitriou, A. et al., 2014, Zhang, Z. et al., 2013, Zhu, D. et al., 2014). Remarkably, in diabetes glomerulus alterations have classically been studied, but recently it has been demonstrated that tubular damage is earlier induced than glomerular injury (Hasegawa, K. et al., 2013) and that tubular impairment sensitizes glomeruli to injury through the tubulo-glomerular feedback (Wang, J. et al., 2018). Moreover, and to the best of our knowledge, despite the pivotal role of proximal tubules on the glucose homeostasis limited works have evaluated the effects of cocoa on the kidney, especially in the renal cortex, in a widely used animal model that mimics human type 2 diabetes, such as Zucker diabetic fatty [ZDF; ZDF/crl-lepr (fa/fa)] rats. Therefore, to understand the potential anti-diabetic activity of cocoa, the aim of this study was to investigate the effects of a cocoa-rich diet on renal morphology, functionality, as well as on the molecular mechanisms connected to the regulation of glucose levels, such as insulin signalling, gluconeogenesis and glucose transporter contents in the renal cortex of ZDF rats. 


\section{Materials and methods}

\subsection{Materials and chemicals.}

Anti-GSK-3 $\alpha / \beta$ and anti-phospho-GSK-3 $\alpha / \beta$ recognizing phosphorylated Ser21/9 of GSK-3, anti-GS and anti-phospho-GS detecting phosphorylated Ser641 of GS, and anti$\beta$-actin were obtained from Cell Signalling Technology (Izasa, Madrid, Spain). AntiIR $\beta$ and anti-phospho-IR $\beta$ recognizing phosphorylated Tyr1150/1151 of IR, antiPEPCK, anti-G-6-Pase and anti-SGLT-2 were purchased from Santa Cruz (sc-711, sc81500, sc-32879 sc-25840 and sc-98975, respectively, Qimigen, Madrid, Spain). AntiGLUT-2 was obtained from Millipore (Madrid, Spain). Glycated haemoglobin (HbA1c) and albumin kits were purchased from Spinreact (BioAnalitica, Madrid, Spain), rat insulin ELISA kit was acquired from Mercodia (AD Bioinstruments, Barcelona, Spain), glucose assay kit was from Sigma (Madrid, Spain), creatinine kit was obtained from Linear Chemicals S.L. (Barcelona, Spain), and the glycogen fluorometric assay kit was from BioVision (Deltaclon, Madrid, Spain). Materials and chemicals for electrophoresis were from BioRad Laboratories S.A. (Madrid, Spain).

\subsection{Cocoa.}

Natural Forastero cocoa powder (a kind gift from Idilia S.L., Barcelona, Spain) was used for this study. A detailed description of this cocoa is given elsewhere (CorderoHerrera, I. et al., 2015, Martín, M.A. et al., 2008).

Diets were prepared from an AIN-93G formulation (Panlab S.L., Barcelona, Spain) providing all nutrients required by adult rats. The $10 \%$ cocoa diet was produced by adding $100 \mathrm{~g} / \mathrm{Kg}$ cocoa to AIN-93G, and the composition of the diets is given elsewhere (Cordero-Herrera, I. et al., 2015). Briefly, AIN-93G formulation lacking cellulose, starch and sucrose was prepared by adding the mentioned ingredients to adjust the 
quantities of carbohydrate and fibre according to the amount of cocoa added. Thus, the $10 \%$ cocoa diet was produced by adding $100 \mathrm{~g} / \mathrm{Kg}$ cocoa to AIN-93G; as a result, the cocoa diet was slightly richer in proteins $(2.2 \%)$ and lipids $(1.1 \%)$, but in order to make both diets isoenergetic, the content in carbohydrate was slightly reduced (4.2\%).

\subsection{Animals and diets.}

Eight male Zucker lean (ZL) rats and sixteen Zucker diabetic fatty [ZDF; ZDF/crl-lepr (fa/fa)] animals were purchased from Charles River Laboratories (L'Arbresle, France) at 9 weeks old. ZDF rats possess a mutation in the leptin receptor and spontaneously develop severe obesity, hyperglycaemia, hyperlipidaemia, and insulin resistance, and are widely used as a model for type 2 diabetes, (Leonard, B.L. et al., 2005).

Animals were caged in groups under controlled conditions $\left(19-23^{\circ} \mathrm{C}, 50-60 \%\right.$ humidity and $12 \mathrm{~h}$ light-dark cycles). After one week of acclimatization, ZDF rats were randomly assorted into two different experimental groups: one group received a standard diet (ZDF-C) and the other group was fed with the cocoa-rich diet (ZDF-Co). ZL rats remained as a unique group and were fed with the standard control diet. Both diets were isocaloric and all animals were provided with food and water ad libitum.

Food intake was monitored daily and animal weight was weekly followed. After 10 weeks, animals were fasted overnight and blood samples were collected for biochemical analysis; serum was separated by centrifugation at $1000 \mathrm{~g}, 10 \mathrm{~min}, 4^{\circ} \mathrm{C}$ for further biochemical analysis. Kidneys were collected, weighted and divided into two samples: one was fixed by immersion in paraformaldehyde (PFA) for histological analysis and the other sample was frozen in liquid $\mathrm{N}_{2}$ and stored at $-80^{\circ} \mathrm{C}$. All animal protocols were approved by the Bioethical Committees from Consejo Superior de Investigaciones Científicas (CSIC, Madrid, Spain) and Universidad Complutense de Madrid (UCM, 
Madrid, Spain), and the regional Committee for Laboratory Animals Welfare (Comunidad de Madrid, Ref. number PROEX-304-15), and were treated according to Institutional Care Instructions (Bioethical Commissions from CSIC and UCM).

\subsection{Biochemical analysis.}

Blood glucose was determined using an Accounted Glucose Analyser (LifeScan España, Madrid, Spain). Serum insulin was analysed with a rat insulin ELISA kit with a detection limit lower than $0.15 \mathrm{ng} / \mathrm{mL}$. HbA1c was measured in blood using a latex turbidimetry kit following the manufacturer's instructions. The minimum detectable amount was $0.1 \%$. Creatinine was analysed with a colorimetric kit following the manufacturer's instructions. Insulin sensitivity was estimated by the Homeostasis model assessment of insulin resistance (HOMA-IR) according to the following formula: [fasting glucose $(\mathrm{mM}) \times$ fasting insulin $(\mathrm{mUI} / \mathrm{L})] / 22.5$, and Homeostasis model assessment of insulin sensitivity (HOMA-IS) according to the following formula: [10000/(fasting glucose $(\mathrm{mg} / \mathrm{dL}) \times$ fasting insulin $(\mathrm{ng} / \mathrm{mL}))]$.

Urine samples were collected over $24 \mathrm{~h}$ by placing the animals in metabolic cages. Urinary glucose and albumin were measured by the glucose oxidase method and a latex turbidimetry kit, respectively, following the manufacturer's instructions. The estimated glomerular filtration rate (eGFR, $\mathrm{mL} / \mathrm{min}$ ) was calculated according to the following formula: [(urine creatinine $(\mathrm{mg} / \mathrm{dL}) \mathrm{x}$ urine volume $(\mathrm{mL} / \mathrm{min})) / \mathrm{serum}$ creatinine $(\mathrm{mg} / \mathrm{dL})]$.

To quantify the renal glycogen content, frozen kidney samples were dissolved in $30 \%$ $\mathrm{KOH}$, boiled and later centrifuged at $12000 \mathrm{~g}$ for $5 \mathrm{~min}$, and glycogen was measured in the supernatants. Glycogen content was measured using a commercial glycogen fluorometric kit (Cordero-Herrera, I. et al., 2014). 


\subsection{Glucose tolerance test (GTT).}

Overnight fasted rats were administered $35 \%$ glucose solution i.p. (2 g/ $\mathrm{Kg}$ of body weight) and blood samples were obtained from the tail vein before the glucose load $(t=0)$ and at 15, 30, 60, 90 and 120 min after glucose administration. Blood glucose levels were measured with a glucometer (LifeScan). Overall changes in glucose during GTT were calculated as the area under the curve (AUC) above the basal levels.

\subsection{Preparation of renal lysates.}

Samples of frozen kidney were homogenized 1:5 (w:v) in extraction buffer [50 mM HEPES (pH 7.5), $150 \mathrm{mM} \mathrm{NaCl}, 1 \mathrm{mM} \mathrm{MgCl} 2,1 \mathrm{mM} \mathrm{CaCl} 2,10 \%$ glycerol, $10 \mathrm{mM}$ $\mathrm{Na}_{4} \mathrm{P}_{2} \mathrm{O}_{7}, 10 \mathrm{mM} \mathrm{NaF}, 2 \mathrm{mM}$ EDTA, $1 \%$ Nonidet P-40, $2 \mathrm{mM} \mathrm{Na}_{3} \mathrm{VO}_{4}, 5 \mu \mathrm{g} / \mathrm{mL}$ leupeptin, $20 \mu \mathrm{g} / \mathrm{mL}$ aprotinin, $2 \mathrm{mM}$ benzamidin and $2 \mathrm{mM}$ phenylmethylsulphonyl fluoride]. Homogenates were centrifuged at $14000 \mathrm{~g}$ for $60 \mathrm{~min}$ and the supernatants were collected, assayed for protein concentration by using the Bradford reagent and stored at $-80^{\circ} \mathrm{C}$ until use for Western blot analyses.

\subsection{Western blot analysis.}

To detect p-(Tyr1150/1151)-IR, IR, GSK3, p-GSK3, GS, p-GS, PEPCK, G-6-Pase, SGLT-2 and GLUT-2, equal amounts of protein were separated by SDS-PAGE and transferred to polyvinylidene difluoride (PVDF) filters (Millipore, Madrid, Spain). Membranes were probed with the corresponding primary antibody followed by incubation with peroxide-conjugated anti-rabbit (GE Healthcare, Madrid, Spain) or antimouse (Sigma, Madrid, Spain) immunoglobulin. Blots were developed with the ECL system (GE Healthcare, Madrid, Spain). Normalization of Western blot was ensured by 
$\beta$-actin and band quantification was carried out with a Scanjet scanner (Hewlett Packard) and the Scion Image software.

\subsection{Histological analysis.}

Left kidney was removed, fixed overnight in $4 \%$ PFA in $0.1 \mathrm{M}$ phosphate buffer $\mathrm{pH} 7.4$ and routinely paraffin embedded. Serial sections $(4 \mu \mathrm{m})$ were mounted on glass slides, hydrated and stained with Haematoxylin and Eosin (H\&E) or periodic-acid-Schiff (PAS), respectively, according to the manufacturer's instructions. To evaluate the histopathological damage, images of stained sections were acquired with magnification at 200x and/or 400x using a digital camera (Leica DFC 320 camera, L’Hospitalet del Llobregat, Spain) attached to a light microscope (Leica DM LB2). Morphometric analysis was carried out with ImageJ v1.52j software (National Institutes of Health: rsb.info.nih.gov/ij) and the colour deconvolution plugin. All slides were examined by two different researchers in a blinded manner. The glomerular and tubular injuries were evaluated as following: glomerular tuft area $\left(\mu \mathrm{m}^{2}\right)$ was defined by tracing the outline of the glomerular tuft as the region of interest (ROI); mesangial expansion was defined as periodic acid-Schiff-positive and nuclei-free area in the mesangial matrix fraction within the glomerular $(\%)$, Bowman's space $\left(\mu \mathrm{m}^{2}\right)$ was calculated by subtracting the glomerular tuft area from the outline of each glomerulus; cortical tubular size representing the tubular dilatation was calculated by tracing the outline of each tubule $\left(\mu \mathrm{m}^{2}\right)$; the tubular epithelial thickness was calculated by subtracting the measured by outlining the apical membrane from outlining each tubular profile $\left(\mu \mathrm{m}^{2}\right)$; cortical hyaline cast area was calculated as the percentage of the cortex occupied by PASpositive stain proteinaceous cast $\left(\mu \mathrm{m}^{2}\right)$ by scoring 10 renal cortical tubules per kidney in 
randomly selected microscopic fields. All areas were calculated from the mean of 10 cortical fields for each kidney or 10 glomeruli cut at the vascular pole in each kidney.

\subsection{Statistics.}

Prior to statistical analysis, data were tested for homogeneity of variances by the test of Levene. For multiple comparisons, one-way ANOVA was followed by the Bonferroni test when variances were homogeneous or by the Tamhane test when variances were not homogeneous. The analysis was followed by a one-way ANOVA. In tables and figures, means not sharing a common superscript letter differ significantly at $P<0.05$. A SPSS version 23.0 program has been used. 


\section{Results}

3.1. Cocoa-rich diet improves body weight, serum glucose and insulin, and HbAlc levels in $Z D F$ rats.

Initial and final body weight, and total food intake were significantly higher in both groups of diabetic rats than in ZL animals (Table 1). Interestingly, the final body weight of animals fed with cocoa diet (ZDF-Co rats) was lower in comparison to ZDF-C group, although still higher than that of ZL group. Moreover, total food intake was lower in ZL rats than in ZDF-C and ZDF-Co animals (Table 1). Both groups of diabetic rats showed greater absolute kidney weight than ZL animals, which demonstrated the typical nephromegaly of the early stage of diabetes, although no differences were observed for the kidney-to body weight ratio among all groups (Table 1).

Glucose and insulin levels were higher in ZDF groups than in ZL rats, and those values were greater in ZDF-C animals than in ZDF-Co rats (Table 2). Additionally, HbA1c levels increased in both diabetic groups when compared to ZL rats, but ZDF-Co animals exhibited lower levels than those of ZDF-C rats.

All these results could suggest that cocoa-rich diet ameliorates hyperglycaemia and hyperinsulinaemia in ZDF rats.

3.2 Cocoa-rich diet improves glucose tolerance and insulin sensitivity in ZDF rats.

To further evaluate the ameliorative effects of the cocoa intake on the diabetic phenotype, GTT was performed. ZDF-C group had impaired glucose tolerance with a higher glucose AUC compared with ZL rats, and the cocoa-rich diet improved the glucose tolerance in ZDF animals (Table 2). In concert, insulin resistance index (HOMA-IR index) increased in ZDF rats vs. ZL, but a significant decrease was observed in ZDF-Co animals in comparison to ZDF-C group (Table 2). Furthermore, insulin sensitivity index (HOMA-IS 
index) was reduced in both ZDF groups when compared to ZL rats, but ZDF-Co animals exhibited higher levels than those of ZDF-C rats (Table 2). Thus, it could be suggested that cocoa-rich diet alleviates glucose intolerance and insulin resistance of ZDF rats.

\subsection{Cocoa-rich diet ameliorates renal dysfunction in $Z D F$ rats.}

To continue with the evaluation of the effects of the cocoa-rich diet on the diabetic rats, serum and urinary parameters related to the renal function were evaluated. Serum creatinine was increased in diabetic rats in comparison to $\mathrm{ZL}$ animals, whereas declined in the rats fed with the cocoa-rich diet (ZDF-Co, Table 2), showing intermediate levels to those of their lean and diabetic littermates. Similarly, in urine both glucose and protein excretion decreased in ZDF-Co animals when compared to ZDF-C rats, although values were still higher than those of the ZL group (Table 2). In addition, eGFR, which showed enhanced levels in ZDF rats when compared to ZL animals, was improved in the ZDF-Co group, displaying lower values than ZDF-C animals, yet higher than those of ZL rats (Table 2). All these changes suggest that cocoa-rich diet might contribute to ameliorate the renal dysfunction in ZDF rats.

3.4. Cocoa-rich diet alleviates renal cortex glomerular and tubular hypertrophy in ZDF rats.

Diabetic kidney disease is accompanied by clinical and morphological alterations (Forbes, J.M. and Cooper, M.E., 2013, Persson, F. and Rossing, P., 2018), and to get further insights into the potential structural changes, histological analyses were performed by $\mathrm{H} \& \mathrm{E}$ and PAS staining in renal cortex sections.

Lean control rats (ZL) demonstrated a normal architecture of the renal corpuscle and renal tubules (Figure 1). In contrast, histopathological examination of the ZDF-C kidneys 
revealed a compensatory glomerular and tubular hypertrophy. In the glomeruli, increased glomerular tuft area, diffused mild mesangial matrix expansion and widening of Bowman's space were observed in ZDF-C when compared to ZL and ZDF-Co groups (Figures 1A-1D). These early hypertrophic processes were restored to comparable values to those of ZL group when ZDF animals were fed with the cocoa-rich diet (Figures 1A1D), demonstrating the reversion of the glomerular hypertrophy to non-diabetic values. In addition, in the renal tubules, ZDF-C rats presented hypertrophic tubular epithelium, dilatation of tubules, increased hyaline casts and accumulation of glycogen in the tubular epithelium (Figures 1A, 1E-1G). However, when compared to ZDF-C animals, ZDF-Co rats showed less-dilated tubules and a narrower tubular epithelium, which resulted in a smaller surface reabsorption (Figures 1A, 1E and 1F). Similarly, the percentage of the area of hyaline cast pinkish amorphous protein within the tubular lumen of ZDF-Co kidney displayed intermediate values in comparison to their lean and diabetic littermates (Figures $1 \mathrm{~A}$ and $1 \mathrm{G}$ ). Altogether it could indicate that cocoa intake could contribute to minimize the adverse morphological renal lesions in ZDF animals.

\subsection{Cocoa-rich diet improves insulin signalling in ZDF rat's renal cortex.}

The role of the insulin signalling pathway in the kidney is thought to contribute to the maintenance of the renal glucose homeostasis (Alvarez-Cilleros, D. et al., 2018, Artunc, F. et al., 2016, Mitrakou, A., 2011, Rahmoune, H. et al., 2005). In view of the obtained results, it was studied whether the cocoa-rich diet modulates the insulin signalling in the renal cortex, and total and phosphorylated levels of main proteins of this pathway were assayed by Western blot.

Tyrosine phosphorylated levels of IR, which are associated to an earlier response to insulin stimulation, were diminished in ZDF-C rats in comparison with ZDF-Co animals, 
which showed similar values to those of ZL group (Figures 2A and 2B). However, there were no significant differences in the content of insulin receptor (IR) among all animal groups (Figures $2 \mathrm{~A}$ and $2 \mathrm{~B}$ )

GSK3 is a rate-limiting enzyme in the glycogen synthesis (Mariappan, M.M. et al., 2008). As shown in Figures $3 \mathrm{~A}$ and 3B, p-GSK3 decreased in ZDF-C rats when compared to ZL and ZDF-Co animals (Figures 3A and 3B). In line with these results, ZDF-C animals presented increased p-GS levels that were restored to ZL values by the cocoa-rich diet in ZDF-Co rats (Figures 3A and 3C). The contents of total GSK3 and GS in the renal cortex were not modified among groups. In addition, an increase in the renal glycogen content in ZDF-C rats was found, whereas ZDF-Co animals showed intermediate values to those of their lean and diabetic littermates (Figures 1A and 3D). Altogether it suggests that the cocoa-rich diet prevents the blockage of the insulin signalling cascade observed in $\mathrm{ZDF}$ rats by modulating main proteins of the insulin pathway from the early steps of this route, contributing to the glucose homeostasis.

\subsection{Cocoa-rich diet modulates rate-limiting proteins of gluconeogenesis and transport of} glucose in the renal cortex of $Z D F$ rats.

In the diabetic renal cortex, an enhanced gluconeogenesis and glucose reabsorption have been reported at the early stage of the disease (Eid, A. et al., 2006, Mitrakou, A., 2011). Thus, to continue with the study of the glucose homeostasis, key proteins involved in renal glucose production and transport, namely PEPCK, G-6-Pase, SGLT-2 and GLUT-2, were evaluated by Western blot in all animal groups.

PEPCK levels increased in the renal cortex of ZDF rats when compared to ZL animals, and this effect was partly reverted in animals receiving the cocoa rich-diet. In this line, values of G-6-Pase increased in ZDF-C animals in comparison to their lean littermates, 
whereas rats fed with the cocoa-rich diet showed comparable levels of G-6-Pase to those of ZL group (Figures 4A and 4B). Additionally, SGLT-2 and GLUT-2 levels were similar in ZL and ZDF-Co animals, but both proteins significantly increased their content in ZDF-C group (Figures 5A-5C). All this suggests that cocoa-rich diet might also contribute to preserve the renal functionality and modulate the glucose homeostasis in ZDF rats. 


\section{Discussion}

Prevention and treatment of the diabetic kidney disease rely on tackling the broad cardiovascular and diabetic factors, being glucotoxicity a major player in this process (Forbes, J.M. and Cooper, M.E., 2013, Persson, F. and Rossing, P., 2018, Thomas, M.C. et al., 2015). In this regard, growing evidence suggests the anti-diabetic activity of cocoa by contributing to control glucose levels (Cordero-Herrera, I. et al., 2015, Costa, C. et al., 2017, Grassi, D. et al., 2008, Jalil, A.-M.-M. et al., 2008, Martín, M.A. et al., 2016, Martín, M.A. et al., 2017, Ruzaidi, A. et al., 2005). However, insufficient studies have been performed at molecular level to support these observations, especially in the kidney, which has been proven to be a key organ for maintaining the glucose homeostasis (Mitrakou, A., 2011, Tiwari, S. et al., 2007, Tiwari, S. et al., 2013).

This work shows that a cocoa-rich diet improves glycaemic control and insulin sensitivity in the renal cortex of ZDF rats. To the best of our knowledge, this is the first in vivo demonstration that a cocoa-rich diet alleviates the diabetic-induced cortical renal dysfunction by reverting the decrease of Tyr-IR phosphorylated levels, the inhibition of GSK3-GS pathway, and the increase of glucose transporters SGLT-2 and GLUT-2 values. Likewise, in the renal cortex, cocoa highly or totally reverts the levels of PEPCK and G-6-Pase to control values, respectively, and protects the renal functionality by restoring the glycogen content and the renal morphological changes.

Cocoa-rich diet decreased the body weight gain without influencing total food intake in comparison to ZDF-C group, as previously shown (Cordero-Herrera, I. et al., 2015, Jalil, A.-M.-M. et al., 2008, Ruzaidi, A. et al., 2005). This effect has been related to the cocoa polyphenolic fraction and its ability to reduce fat adipose tissue. In this line, absolute kidney weights increased in the diabetic animals (nephromegaly), whereas kidney/body weight ratios were similar among all animal groups, indicating an early 
stage of the diabetic kidney disease (Baynes, J. and Murray, D.B., 2009, Nakayama, T. et al., 2010).

Diabetes is characterized by hyperglycaemia, hyperinsulinaemia, glucose intolerance (AUC), insulin resistance (HOMA-IR), enhanced values of HbA1c, together with reduced insulin sensitivity (HOMA-IS) (Cordero-Herrera, I. et al., 2015, Muthenna, P. et al., 2014). All these metabolic alterations were observed in ZDF-C animals, which demonstrate their diabetic stage (Table 2). However, all these parameters were highly reverted to control values (ZL group) in rats receiving the cocoa-rich diet, indicating that cocoa improves glucose metabolism, as previously shown in diabetic rodents fed with cocoa, oligomeric cocoa procyanidins or different cocoa extracts (Cordero-Herrera, I. et al., 2015, Dorenkott, M.R. et al., 2014, Jalil, A.-M.-M. et al., 2008, Ruzaidi, A. et al., 2005). In addition, during diabetes there are usually changes in the renal structure and function, which have been mainly related to the glucotoxicity (Forbes, J.M. and Cooper, M.E., 2013, Persson, F. and Rossing, P., 2018). Indeed, the early diabetic kidney undergoes hyperfunction and hypertrophy (Forbes, J.M. and Cooper, M.E., 2013). Thus, the hyperfiltration explains the high levels of serum creatinine, the presence of albumin and glucose in the urine (albuminuria and glycosuria, respectively) and therefore, the enhanced values of eGFR in ZDF-C rats (Table 2). Moreover, these clinical alterations were accompanied by morphological changes in the renal cortex associated to the hyperfiltration and mainly related to the high levels of glucose. Actually, histological kidney lesions in the ZDF-C rats observed in the present study are in agreement with an early stage of diabetes (classification criteria IIa, according to the classification system introduced by Renal Pathology Society) (Jain, M., 2012). Among these structural alterations, ZDF-C animals showed glomerular hypertrophy, mild mesangial matrix expansion and dilated Bowman's space, as well as tubular 
hypertrophy with increased tubular epithelium thickness, dilatation of the tubules, and increased hyaline casts formation (Figure 1). All these hypertrophic changes have been connected to an augmented kidney weight, nephromegaly and elevation in serum creatinine (Cohen, M.P. et al., 1996). Indeed, the degree of proteinuria displayed by diabetic rats is directly correlated with the hyaline droplet formation within the tubular lumen and, also reflects an enhanced protein reabsorption (Forbes, J.M. and Cooper, M.E., 2013). It has also been reported that hyperfiltration related to glomerular injury is secondary to increased proximal tubular resorption of glucose (Hasegawa, K. et al., 2013). Moreover, due to the growth of the tubule more filtrate is reabsorbed, which leads to increased GFR by a feedback-loop from the tubules (Forbes, J.M. and Cooper, M.E., 2013). Thus, the compensatory functional and structural hypertrophy (glomeruli and associated tubules) showed in ZDF-C animals is a consequence of attempting to maintain filtration and reabsorption within the normal range during the early stage of diabetes. Nevertheless, all these changes were reverted by feeding ZDF rats with cocoa (ZDF-Co). In agreement, in diabetic hypertensive rats administration of cocoa contributed to prevent the glomerular mesangial expansion (Papadimitriou, A. et al., 2014). Similarly, foods rich in flavanols or pure flavanols, such as green tea, Juglans regia L. leaf powder, catechin, and procyanidin B2 from grape seed and cinnamon have demonstrated protective effects against high glucose-induced structural and functional alterations in kidneys of different diabetic rodent models (Mollica, A. et al., 2017, Muthenna, P. et al., 2014, Renno, W.M. et al., 2008, Zhang, Z. et al., 2013, Zhu, D. et al., 2014). All this supports the fact that most of renal alterations can be reversed at an early stage by a good glycaemic control (Thomas, M.C. et al., 2015).

Renal tubules play a crucial role in the glucose homeostasis and their dysfunction constitutes an early pathophysiological alteration that correlates with an impaired renal 
function in the diabetic kidney disease (Forbes, J.M. and Cooper, M.E., 2013, Mollica, A. et al., 2017). Indeed, chronic hyperglycaemia, i.e. glucotoxicity, disrupt the glucose homeostasis, also in the kidney, and lead to the alteration of metabolic pathways, such as gluconeogenesis, glucose uptake and insulin signalling (Artunc, F. et al., 2016, Mitrakou, A., 2011, Rahmoune, H. et al., 2005). Thus, during a situation of chronic high levels of glucose the renal insulin signalling is impaired and the modulation of IR, which is essential for recruiting and activating downstream pathways, is damaged, being diminished the phosphorylated levels of IR in particular tyrosine residues (Tiwari, S. et al., 2007), as shown in the present study. However, the cocoa-rich diet prevented this critical aspect for the development of insulin resistance in ZDF rats. In agreement with this, we have recently reported that epicatechin, the main flavanol of cocoa, attenuated insulin resistance by preventing the decrease of IR tyrosine phosphorylation induced by high levels of glucose in renal tubular NRK-52E cells (Álvarez-Cilleros, A. et al., 2018). Similarly, the flavonoids quercetin and rutin alleviated insulin resistance by increasing IR tyrosine phosphorylation in the kidney of fructose-fed rats (Hu, Q.-H. et al., 2012).

Following the insulin signalling route downstream, the activation of IR leads to the inhibition of GSK3 (enhanced phosphorylation at Ser9), which subsequently phosphorylates and inactivates GS (Nystrom, F.H. and Quon, M.J., 1999). In this regard, GSK3 inhibition has been related to the amelioration of the injury induced by diabetes in kidneys (Mariappan, M.M. et al., 2008). In agreement, the renal cortex of ZDF-C rats showed decreased p-GSK3, and increased GS phosphorylated levels, while enhanced glycogen content compared to ZL animals, as previously shown in the liver of ZDF animals (Cordero-Herrera, I. et al., 2015, Leonard, B.L. et al., 2005). These effects were highly reverted when animals were fed with the cocoa-rich diet. In line with these 
results, grape seed procyanidin B2 highly restored p-GSK3 levels to control values in the kidney of diabetic mice, and this was associated to a protective effect in the development of the diabetic nephropathy (Zhang, Z. et al., 2013). Similarly, in Zucker rats a specific GSK3 inhibitor enhanced oral glucose tolerance and insulin sensitivity (Dokken, B.B. and Henriksen, E.J., 2006), and we have recently demonstrated that epicatechin improved insulin signalling-mediated GSK3 activation in high glucosetreated NRK-52E cells (Álvarez-Cilleros, A. et al., 2018). Interestingly, administration of green tea prevented glycogen accumulation in the kidney tubules of diabetic rats (Renno, W.M. et al., 2008). All together indicates that cocoa could improve renal insulin resistance and contribute to the glucose homeostasis by inhibiting GSK3 activation and preventing the enhanced glycogen content in the renal cortex of ZDF rats. Renal gluconeogenesis occurs in the cortex, and also represents an important mechanism for the regulation of the glucose homeostasis (Mitrakou, A., 2011). Indeed, in the renal cortex of diabetic patients and rats enhanced levels of gluconeogenic PEPCK and G-6-Pase enzymes have been reported (Eid, A. et al., 2006, Gatica, R. et al., 2012, Tiwari, S. et al., 2013). Thus, downregulation of both enzymes, as observed in cocoa-fed ZDF rats when compared to ZDF-C group, could contribute to inhibit the renal gluconeogenesis, and then to diminish the glycaemia of diabetic animals, as previously shown for areca nut procyanidins, cinnamaldehyde, and vanadium compounds alone or in combination with Trigonella foenum graecum seed powder (Anand, P. et al., 2010, Huang, P.L. et al., 2013, Marzban, L. et al., 2002, Mohamad, S. et al., 2004). In this line, downregulation of IR in renal proximal tubule in insulinresistant rats has been suggested to contribute to maintain high glucose levels through enhanced gluconeogenesis (Tiwari, S. et al., 2013). Therefore, all these results suggest that cocoa might also contribute to diminish the hyperglycaemia via the restoration of 
gluconeogenic PEPCK and G-6-Pase enzymes in ZDF rats, playing insulin signalling (IR) a physiological role in this process.

In the diabetic renal cortex, reabsorbed glucose by SGLT-2 is released into the circulation through GLUT-2, leading to upregulated levels of both transporters at the early stage of the disease, and contributing to worsen the situation of glucotoxicity and insulin resistance (Dion, F. et al., 2017, Mitrakou, A., 2011). These alterations in glucose reabsorption influenced the glucose homeostasis, and led to the accumulation of glycogen in the renal tubules, among other changes (Mitrakou, A., 2011, Renno, W.M. et al., 2008). In addition, albumin has also been reported to upregulate the expression of SGLT-2 in diabetes (Mitrakou, A., 2011). In agreement with all of the above, enhanced contents of SGLT-2 and GLUT-2 were detected in diabetic ZDF-C rats, whereas the transporter levels returned to control values (ZL group) in ZDF animals fed with cocoa. In this line, natural substances, such as phlorizin and resveratrol contribute to restore SGLT-2 and GLUT-2 levels in diabetic rodents (Brouwers, B. et al., 2013, Yonamine, C.Y. et al., 2016). In addition, the decreased proximal glucose reabsorption, as consequence of diminished SGLT-2 levels in ZDF-Co rats, might restore the normal tubulo-glomerular feedback mechanism, and contribute to reduce the glomerular hyperfiltration. Also, the inhibition of glucose reabsorption induced by cocoa might be able to attenuate the renal hypertrophy associated with the experimental diabetes. Therefore, the improved glucose homeostasis and insulin sensitivity of ZDF-Co animals could be associated to the modulatory effect of the cocoa-rich diet on SGLT-2 and GLUT-2 contents.

\section{Conclusions}


A cocoa-rich diet improves the glucose homeostasis and insulin resistance in ZDF rats. Cocoa alleviates high glucose levels through the restoration of renal glucose transporters (SGLT-2 and GLUT-2) levels and glycogen content, as well as through the modulation of key gluconeogenic enzymes, protecting the renal structure and functionality. Likewise, cocoa improves glucotoxicity-induced insulin resistance by increasing IR-Tyr phosphorylation and activating the GSK3/GS pathway. This study provides for the first time novel mechanistic insights into the preventive effects of cocoa on renal insulin resistance in a situation of glucotoxicity and, despite further efforts are needed, it could be suggested that cocoa might contribute to reduce the progression of renal disease in type 2 diabetic patients. 


\section{Conflict of interest}

The authors declare that there are no conflicts of interest.

\section{Acknowledgments}

This work was supported by the grant AGL2015-67087-R (MINECO/FEDER, UE)

from the Spanish Ministry of Science and Innovation (MINECO). D. Álvarez-Cilleros is a FPI fellow from the predoctoral program of MINECO (BES-2016-076721). 


\section{References}

Álvarez-Cilleros, A., Martín, M.A. and Ramos, S., 2018. Protective effects of (-)epicatechin and the colonic metabolite 3,4-dihydroxyphenylacetic acid against glucotoxicity-induced insulin signalling blockade and altered glucose uptake and production in renal tubular NRK-52E cells. Food Chem Toxicol. 120, 119-128.

Alvarez-Cilleros, D., Ramos, S., Goya, L. and Martín, M.A., 2018. Colonic metabolites from flavanols stimulate nitric oxide production in human endothelial cells and protect against oxidative stress-induced toxicity and endothelial dysfunction. Food Chem Toxicol. 115, 88-97.

Anand, P., Murali, K.Y., Tandon, V., Murthy, P.S. and Chandra, R., 2010. Insulinotropic effect of cinnamaldehyde on transcriptional regulation of pyruvate kinase, phosphoenolpyruvate carboxykinase, and GLUT4 translocation in experimental diabetic rats. Chem Biol Interact. 186, 72-81.

Artunc, F., Schleicher, E., Weigert, C., Fritsche, A., Stefan, N. and Häring, H.U., 2016. The impact of insulin resistance on the kidney and vasculature. Nat Rev Nephrol. 12, 721-737.

Baynes, J. and Murray, D.B., 2009. Cardiac and renal function are progressively impaired with aging in Zucker diabetic fatty type II diabetic rats. Oxid Med Cell Longev. 2, 328-334.

Brouwers, B., Pruniau, V.P., Cauwelier, E.J., Schuit, F., Lerut, E., Ectors, N., Declercq, J. and Creemers, J.W., 2013. Phlorizin pretreatment reduces acute renal toxicity in a mouse model for diabetic nephropathy. J Biol Chem. 288, 27200-27207.

Cohen, M.P., Clements, R.S., Cohen, J.A. and Shearman, C.W., 1996. Prevention of decline in renal function in the diabetic $\mathrm{db} / \mathrm{db}$ mouse. Diabetologia. 39, 270-274. 
Cordero-Herrera, I., Martín, M.A., Escrivá, F., Álvarez, C., Goya, L. and Ramos, S., 2015. Cocoa-rich diet ameliorates hepatic insulin resistance by modulating insulin signaling and glucose homeostasis in Zucker diabetic fatty rats. J Nutr Biochem. 26, 704-712.

Cordero-Herrera, I., Martín, M.A., Goya, L. and Ramos, S., 2014. Cocoa flavonoids attenuate high glucose-induced insulin signalling blockade and modulate glucose uptake and production in human HepG2 cells. Food Chem Toxicol. 64, 10-19.

Costa, C., Tsatsakis, A., Mamoulakis, C., Teodoro, M., Briguglio, G., Caruso, E., Tsoukalas, D., Margina, D., Dardiotis, E., Kouretas, D. and Fenga, C., 2017. Current evidence on the effect of dietary polyphenols intake on chronic diseases. Food Chem Toxicol. 110, 286-299.

Dion, F., Dumayne , C., Henley, N., Beauchemin, S., Arias, E.B., Leblond, F.A., Lesage, S., Lefrançois, S., Cartee, G.D. and Pichette , V., 2017. Mechanism of insulin resistance in a rat model of kidney disease and the risk of developing type 2 diabetes. PLoS One. 12, e0176650.

Dokken, B.B. and Henriksen, E.J., 2006. Chronic selective glycogen synthase kinase-3 inhibition enhances glucose disposal and muscle insulin action in prediabetic obese Zucker rats. Am J Physiol Endocrinol Metab. 291, E207-E213.

Dorenkott, M.R., Griffin, L.E., Goodrich, K.M., Thompson-Witrick, K.A., Fundaro, G., Ye, L., Stevens, J.R., Ali, M., O’Keefe, S.F., Hulver, M.W. and Neilson, A.P., 2014. Oligomeric cocoa procyanidins possess enhanced bioactivity compared to monomeric and polymeric cocoa procyanidins for preventing the development of obesity, insulin resistance, and impaired glucose tolerance during high-fat feeding. J Agric Food Chem. 62, 2216-2227. 
Eid, A., Bodin, S., Ferrier, B., Delage, H., Boghossian, M., Martin, M., Baverel, G. and Conjard, A., 2006. Intrinsic gluconeogenesis is enhanced in renal proximal tubules of Zucker diabetic fatty rats. J Am Soc Nephrol. 17, 398-405.

Forbes, J.M. and Cooper, M.E., 2013. Mechanisms of diabetic complications. Physiol Rev. 93, 137-188.

Gatica, R., Bertinat, R., Silva, P., Carpio, D., Ramírez, M.J., Slebe, J.C., San Martín, R., Nualart, F., Campistol, J.M., Caelles, C. and Yáñez, A.J., 2012. Altered expression and localization of the insulin receptor in proximal tubule cells from human and rat diabetic kidney. J Cell Biochem. 114, 639-649.

Grassi, D., Desideri, G., Necozione, S., Lippi, C., Casale, R., Properzi, G., Blumberg, J.B. and Ferri, C., 2008. Blood pressure is reduced and insulin sensitivity increased in glucose-intolerant, hypertensive subjects after 15 days of consuming high-polyphenol dark chocolate. J Nutr. 138, 1671-1676.

Hasegawa, K., Wakino, S., Simic, P., Sakamaki, Y., Minakuchi, H., Fujimura, K., Hosoya, K., Komatsu, M., Kaneko, Y., Kanda, T., Kubota, E., Tokuyama, H., Hayashi, K., Guarente, L. and Itoh, H., 2013. Renal tubular Sirt1 attenuates diabetic albuminuria by epigenetically suppressing Claudin-1 overexpression in podocytes. Nat Med. 19, 1496-1504.

Hu, Q.-H., Zhang, X., Pan, Y., Li, Y.-C. and Kong, L.-D., 2012. Allopurinol, quercetin and rutin ameliorate renal NLRP3 inflammasome activation and lipid accumulation in fructose-fed rats. Biochem Pharmacol. 84, 113-125.

Huang, P.L., Chi, C.W. and Liu, T.Y., 2013. Areca nut procyanidins ameliorate streptozocin-induced hyperglycemia by regulating gluconeogenesis. Food Chem Toxicol. 55, 137-143. 
Igarashi, K., Honma, K., Yoshinari, O., Nanjo, F. and Hara, Y., 2007. Effects of dietary catechins on glucose tolerance, blood pressure and oxidative status in GotoKakizaki rats. J Nutr Sci Vitaminol. 53, 496-500.

Jain, M., 2012. Histopathological changes in diabetic kidney disease. Clinical Queries: Nephrology. 102, 127-133.

Jalil, A.-M.-M., Ismail, A., Pei, C.-P., Hamid, M. and Kamaruddin, S.-H.-S., 2008. Effects of cocoa extract on glucometabolism, oxidative stress, and antioxidant enzymes in obese-diabetic (ob-db) rats. J Agric Food Chem. 56, 7877-7884.

Kim, Y., Keogh, J.B. and Clifton, P.M., 2016. Polyphenols and glycemic control. Nutrients. 8, 17.

Leonard, B.L., Watson, R.N., Loomes, K.M., Phillips, A.R.J. and Cooper, G.J., 2005. Insulin resistance in the Zucker diabetic fatty rat: a metabolic characterisation of obese and lean phenotypes. Acta Diabetol. 42, 162-170.

Mariappan, M.M., Shetty, M., Sataranatarajan, K., Choudhury, G.G. and Kasinath, B.S., 2008. Glycogen synthase kinase $3 \beta$ is a novel regulator of high glucose- and high insulin-induced extracellular matrix protein synthesis in renal proximal tubular epithelial cells J Biol Chem. 283, 30566-30575.

Martín, M.A., Goya, L. and Ramos, S., 2016. Antidiabetic actions of cocoa flavanols. Mol Nutr Food Res. 60, 1756-1769.

Martín, M.A., Goya, L. and Ramos, S., 2017. Protective effects of tea, red wine and cocoa in diabetes. Evidences from human studies. Food Chem Toxicol. 109, 302-314.

Martín, M.A., Ramos, S., Mateos, R., Granado-Serrano, A.B., Izquierdo-Pulido, M., Bravo, L. and Goya, L., 2008. Protection of human HepG2 cells against oxidative stress by cocoa phenolic extract. J Agric Food Chem. 56, 7765-7772. 
Marzban, L., Rahimian, R., Brownsey, R.W. and McNeill, J.H., 2002. Mechanisms by which bis(maltolato)oxovanadium(IV) normalizes phosphoenolpyruvate carboxykinase and glucose-6-phosphatase expression in streptozotocin-diabetic rats in vivo. Endocrinology. 143, 4636-4645.

Mitrakou, A., 2011. Kidney: its impact on glucose homeostasis and hormonal regulation. Diabetes Res Clin Pract. 93S, S66-S72.

Mohamad, S., Taha, A., Bamezai, R.N.K., Basir, S.F. and Baquer, N.Z., 2004. Lower doses of vanadate in combination with trigonella restore altered carbohydrate metabolism and antioxidant status in alloxan-diabetic rats. Clin Chim Acta. 342, $105-114$

Mollica, A., Zengin, G., Locatelli, M., Stefanucci, A., Macedonio, G., Bellagamba, G., Onaolapo, O., Onaolapo , A., Azeez, F., Ayileka, A. and Novellino, E., 2017. An assessment of the nutraceutical potential of Juglans regia L. leaf powder in diabetic rats. Food Chem Toxicol. 107, 554-564.

Muthenna, P., Raghu, G., Kumar, P.A., Surekha, M.V. and Reddy, G.B., 2014. Effect of cinnamon and its procyanidin-B2 enriched fraction on diabetic nephropathy in rats. Chem Biol Interact. 222, 68-76.

Nakayama, T., Kosugi, T., Gersch, M., Connor, T., Sanchez-Lozada, L.G., Lanaspa, M.A., Roncal, C., Perez-Pozo, S.E., Johnson, R.J. and Nakagawa, T., 2010. Dietary fructose causes tubulointerstitial injury in the normal rat kidney. Am J Physiol Renal Physiol. 298, F712-F720.

Nystrom, F.H. and Quon, M.J., 1999. Insulin signalling: metabolic pathways and mechanisms for specificity. Cell Signal. 11, 563-574.

Papadimitriou, A., Peixoto, E.B.M.I., Silva, K.C., Lopes de Faria, J.M. and Lopes de Faria, J.B., 2014. Increase in AMPK brought about by cocoa is renoprotective in 
experimental diabetes mellitus by reducing NOX4/TGF $\beta-1$ signaling. J Nutr Biochem. 25, 773-784.

Persson, F. and Rossing, P., 2018. Diagnosis of diabetic kidney disease: state of the art and future perspective. Kidney Int Suppl. 8, 2-7.

Rahmoune, H., Thompson, P.W., Ward, J.M., Smith, C.D., Hong, G. and Brown, J., 2005. Glucose transporters in human renal proximal tubular cells isolated from the urine of patients with non-insulin-dependent diabetes. Diabetes. 54, 32473234.

Renno, W.M., Abdeen, S., Alkhalaf, M. and Asfar, S., 2008. Effect of green tea on kidney tubules of diabetic rats. Br J Nutr. 100, 652-659.

Ruzaidi, A., Amin, I., Nawalyah, A.G., Hamid, M. and Faizul, H.A., 2005. The effect of Malaysian cocoa extract on glucose levels and lipid profiles in diabetic rats. J Ethnopharmacol. 98, 55-60.

Thomas, M.C., Brownlee, M., Susztak, K., Sharma, K., Jandeleit-Dahm, K.A.M., Zoungas, S., Rossing, P., Groop, P.-H. and Cooper, M.E., 2015. Diabetic kidney disease. Nat Rev Disease primer. 15018, doi:10.1038/nrdp.2015.18,

Tiwari, S., Halagappa, V.K., Riazi, S., Hu, X. and Ecelbarger, C.A., 2007. Reduced expression of insulin receptors in the kidneys of insulin-resistant rats. J Am Soc Nephrol. 18, 2661-2671.

Tiwari, S., Singh, R.S., Li, L., Tsukerman, S., Godbole, M., Pandey, G. and Ecelbarger, C.M., 2013. Deletion of the insulin receptor in the proximal tubule promotes hyperglycemia. J Am Soc Nephrol. 24, 1209-1214.

Vinson, J.A., Proch, J., Bose, P., Muchler, S., Taffera, P., Shutta, D., Samman, N. and Agbor, G.A.C., 2006. Chocolate is a powerful ex vivo and in vitro antioxidant, antiatherosclerotic agent in an animal model, and a significant contributor to 
antioxidants in the European and American diets. J Agric Food Chem. 54, 80718076.

Wang, J., Zhong, J., Yang, H.-C. and Fogo, A.B., 2018. Cross talk from tubules to glomeruli. Toxicol Pathol. 46, 944-948.

Winocour, P.H., 2018. Diabetes and chronic kidney disease: an increasingly common multi- morbid disease in need of a paradigm shift in care. Diabetic Med. 35, $300-305$

Yonamine, C.Y., Pinheiro-Machado, E., Michalani, M.L., Soares-Freitas, H., Okamoto, M.M., Corrêa-Giannella, M.L. and Machado, U.F., 2016. Resveratrol improves glycemic control in insulin-treated diabetic rats: participation of the hepatic territory. Nutr \& Metab. 13, 44, DOI 10.1186/s12986-016-0103-0.

Zhang, Z., Li, B.-Y., Li, X.-L., Cheng, M., Yu, F., Lu, W.-D., Cai, Q., Wang, J.-F., Zhou, R.-H., Gao, H.-Q. and Shen, L., 2013. Proteomic analysis of kidney and protective effects of grape seed procyanidin $\mathrm{B} 2 \mathrm{in} \mathrm{db} / \mathrm{db}$ mice indicate MFG-E8 as a key molecule in the development of diabetic nephropathy. Biochem Biophys Acta. 1832, 805-813.

Zhu, D., Wang, L., Zhou, Q., Yan, S., Li, Z., Sheng, J. and Zhang, W., 2014. (+)Catechin ameliorates diabetic nephropathy by trapping methylglyoxal in type 2 diabetic mice. Mol Nutr Food Res. 58, 2249-2260. 


\section{Legends to figures}

Figure 1. Histological analyses of renal cortex in Zucker lean (ZL), Zucker Diabetic rats fed with control diet (ZDF-C) and Zucker Diabetic rats fed with cocoa diet (ZDFCo). (A) Representative sections of renal cortex stained with H\&E and PAS (scale bar= $20 \mu \mathrm{m})$. Green arrowheads indicate dilated tubules. Yellow arrowheads indicate PASpositive hyaline casts in distal tubules. (B) Glomerular tuft area. (C) Mesangial area. (D) Bowman's space area. (E) Cortical tubular area. (F) Cortical tubular thickness. (G) Cortical hyaline cast area. Data are shown as mean \pm SD. $n=6-8$. Means not sharing a common letter differ significantly, $P<0.05$.

Figure 2. Effect of the cocoa-rich diet on phosphorylated and total levels of IR in the renal cortex of ZDF rats. (A) Bands of representative experiments. (B) Densitometric quantification of p-IR (Tyr) and IR. Values are expressed as a percentage relative to ZL rats. Equal loading of Western blots was ensured by $\beta$-actin (n=6-8). Means not sharing a common letter differ $(P<0.05)$. Different styles of letters (bold and normal) have been used for each parameter (p-IR and IR levels, respectively) depicted within the same graph.

Figure 3. Effect of the cocoa-rich diet on tyrosine phosphorylated and total levels of GSK3 $\beta$ and GS, and glycogen content in the renal cortex of ZDF rats. (A) Bands of representative blots. Percentage data of (B) p-GSK3/GSK3 and (C) pGS/GS ratios relative to ZL group. Values are expressed as means $\pm \mathrm{SD}, \mathrm{n}=6-8$. Equal loading of Western blots was ensured by $\beta$-actin. (D) Glycogen content expressed as percent of 
control are means \pm SD of 6-8 different samples per animal group. Means not sharing a common letter differ $(P<0.05)$.

Figure 4. Effect of the cocoa-rich diet on total levels of PEPCK and G-6-Pase in the renal cortex of $\mathrm{ZDF}$ rats. (A) Bands of representative experiments. Densitometric quantification of (B) PEPCK and (C) G-6-Pase. Values are expressed as a percentage relative to ZL animal group. Equal loading of Western blots was ensured by $\beta$-actin (n=6-8). Means not sharing a common letter differ $(P<0.05)$.

Figure 5. Effect of the cocoa-rich diet on levels of SGLT-2 and GLUT-2 in the renal cortex of ZDF rats. (A) Bands of representative experiments. Percentage data of (B) SGLT-2 and (C) GLUT-2 relative to ZL animals (means $\pm S D, n=6-8$ ). Equal loading of Western blots was ensured by $\beta$-actin. Means not sharing a common letter differ significantly $(P<0.05)$. 
Table 1. Body weight data, food intake and kidney weights of rats fed with standard (ZL and ZDF-C) and cocoa-rich (ZDF-Co) diets*.

ZL ZDF-C $\quad$ ZDF-Co

Initial body weight (g)

$$
\begin{array}{ccc}
228.00 \pm 15.00^{\mathrm{a}} & 282.00 \pm 11.00^{\mathrm{b}} & 282.00 \pm 13.00^{\mathrm{b}} \\
329.00 \pm 4.00^{\mathrm{a}} & 444.00 \pm 8.00^{\mathrm{c}} & 409.00 \pm 7.00^{\mathrm{b}} \\
101.00 \pm 12.00^{\mathrm{a}} & 162.00 \pm 9.00^{\mathrm{c}} & 127.00 \pm 17.00^{\mathrm{b}} \\
1239.00 \pm 53.00^{\mathrm{a}} & 1875.00 \pm 63.00^{\mathrm{b}} & 1872.00 \pm 79.00^{\mathrm{b}}
\end{array}
$$

Final body weight (g)

Body weight gain (g in 10 weeks)

Total food Intake (g in 10 weeks)

Kidney weight $(\mathrm{g})$

$1.12 \pm 0.11^{\mathrm{a}}$

$1.56 \pm 0.19^{b}$

$1.44 \pm 0.11^{b}$

Kidney-to body weight ratio (x 100)

$0.34 \pm 0.03^{\mathrm{a}}$

$0.35 \pm 0.04^{\mathrm{a}}$

$0.35 \pm 0.03^{\mathrm{a}}$

* Data represent the means \pm SD. Means in a row not sharing a common superscript letter differ, $P<0.05$. 
Table 2. Biochemical parameters related to the metabolism and renal function of rats fed with standard (ZL and ZDF) and cocoa-rich (ZDF-Co) diets*.

\section{ZL}

ZDF-C

ZDF-Co

\section{Serum}

Glucose (mmol/L)

$$
5.23 \pm 0.14^{\mathrm{a}}
$$

$13.82 \pm 0.50^{\mathrm{c}}$

$7.37 \pm 0.28^{\mathrm{b}}$

Insulin $(\mathrm{ng} / \mathrm{mL})$

$$
0.41 \pm 0.01^{\mathrm{a}}
$$

$4.52 \pm 0.19^{c}$

$1.25 \pm 0.08^{\mathrm{b}}$

$\operatorname{HbA1c}(\%)$

$$
4.40 \pm 0.12^{\mathrm{a}}
$$

$10.67 \pm 0.58^{\mathrm{c}}$

$6.10 \pm 0.24^{b}$

AUC glucose (mM/min)

$$
1838.00 \pm 80.00^{\mathrm{a}} \quad 4242.00 \pm 566.00^{\mathrm{c}} \quad 3141.00 \pm 205.00^{\mathrm{b}}
$$

HOMA-IR

$$
2.67 \pm 0.12^{\mathrm{a}}
$$

$90.96 \pm 14.25^{\mathrm{c}}$

$12.71 \pm 0.67^{b}$

HOMA-IS

$$
273.39 \pm 22.22^{\mathrm{c}}
$$

$$
7.95 \pm 1.39^{\mathrm{a}}
$$

$55.81 \pm 2.98^{\mathrm{b}}$

Creatinine (mg/dL)

$$
0.18 \pm 0.01^{\mathrm{a}}
$$

$0.26+ \pm 0.02^{\mathrm{c}}$

$0.20 \pm 0.02^{b}$

\section{Urine}

$$
0.63 \pm 0.07^{\mathrm{a}} \quad 67.95 \pm 8.29^{\mathrm{c}} \quad 48.69 \pm 6.38^{\mathrm{b}}
$$

Glucose (mmol/24 h)

$$
2.59 \pm 0.52^{\mathrm{a}} \quad 46.40 \pm 3.26^{\mathrm{c}} \quad 38.87 \pm 1.76^{\mathrm{b}}
$$

Albumin (mg/24 h)

$$
0.41 \pm 0.06^{\mathrm{a}} \quad 0.98 \pm 0.15^{\mathrm{c}} \quad 0.56 \pm 0.05^{\mathrm{b}}
$$

eGFR (mL/min)

\footnotetext{
* Data represent the means \pm SD. Means in a row not sharing a common superscript letter differ, $P<0.05$.
} 
Figure 1

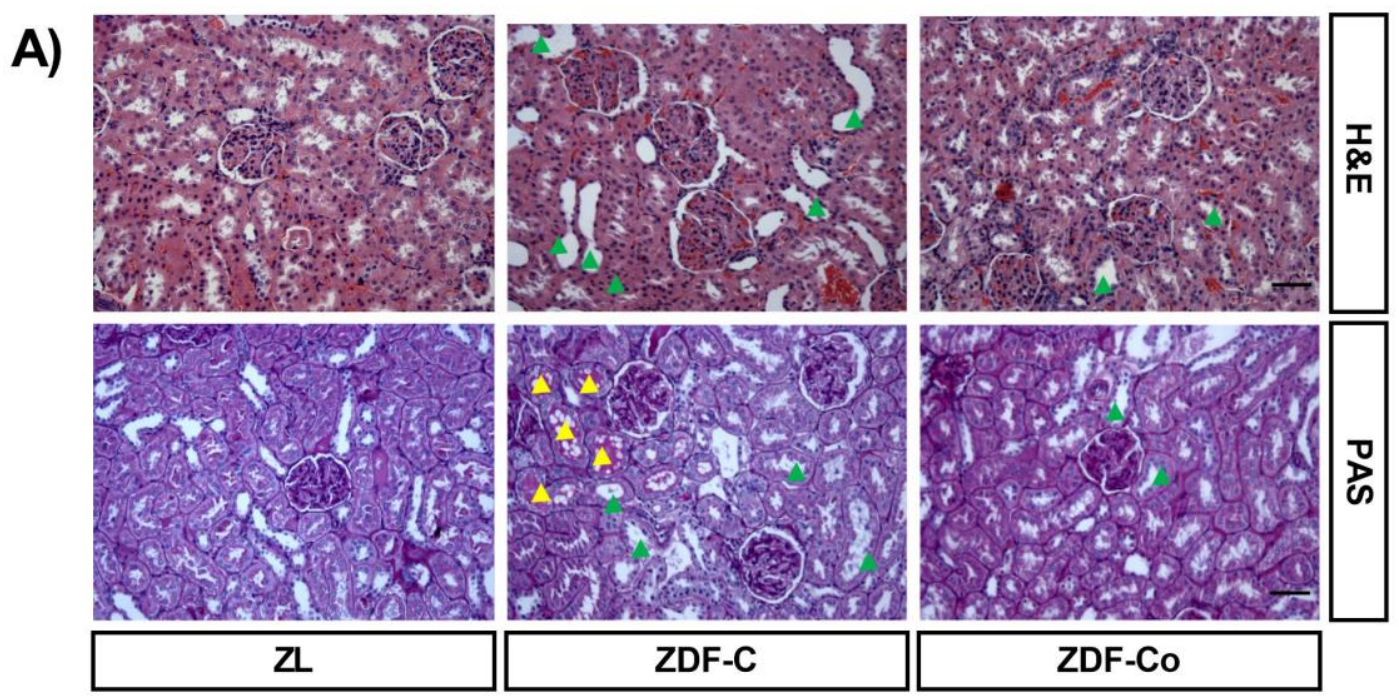

B)

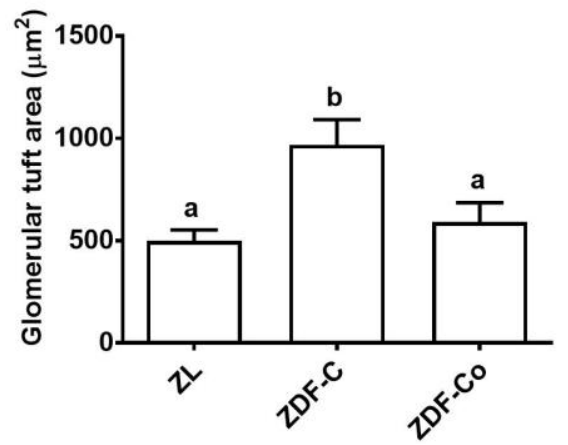

D)

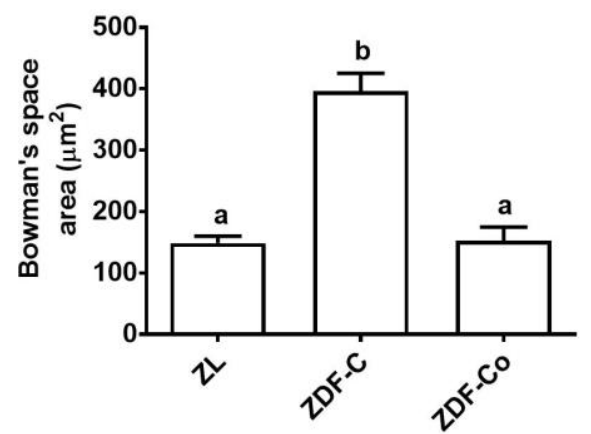

F)

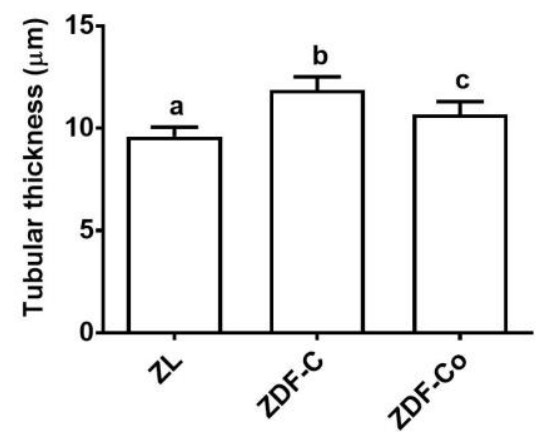

C)

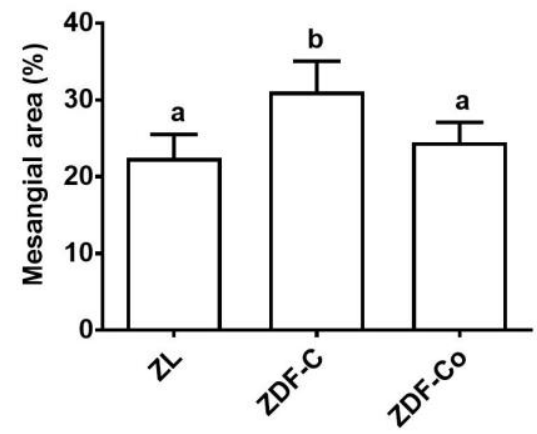

E)

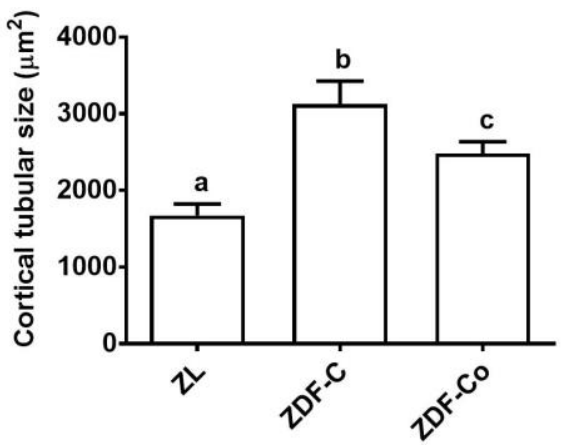

G)

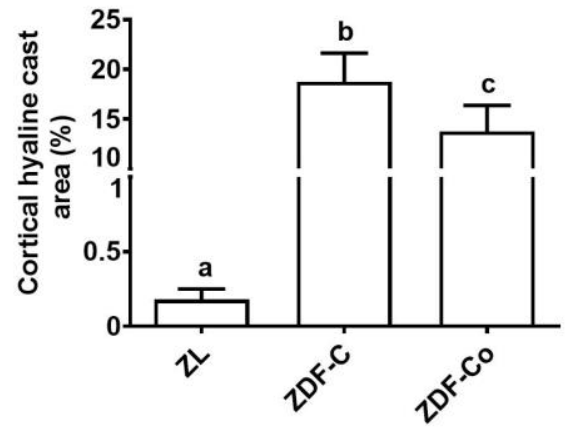


Figure 2

A)

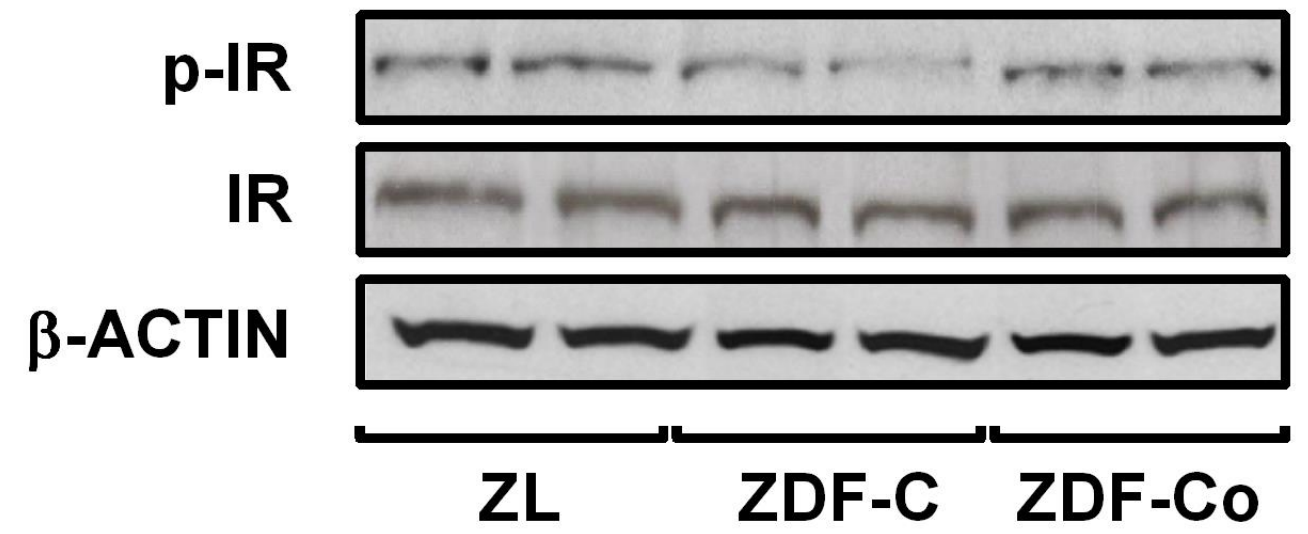

B)

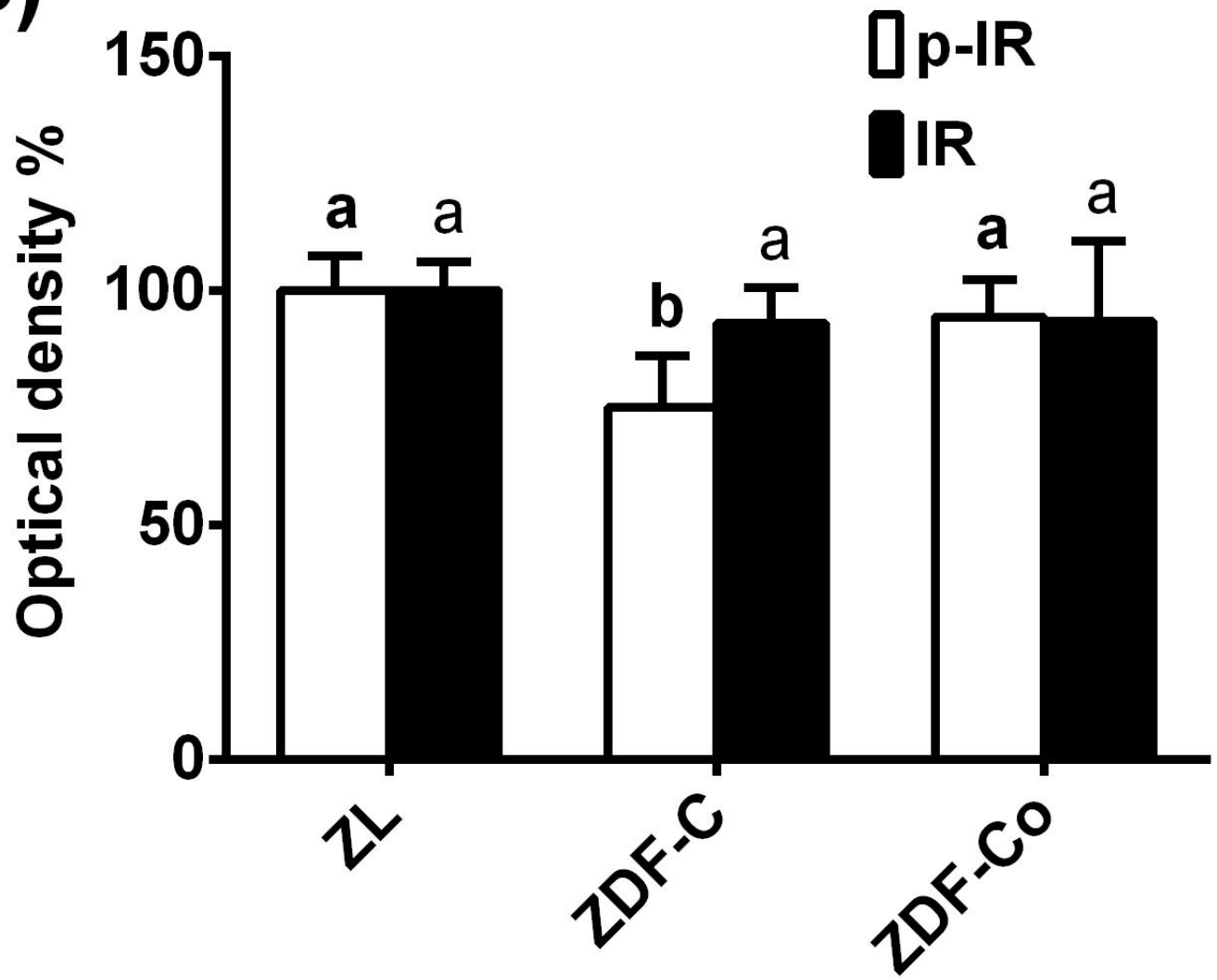


Figure 3

A)

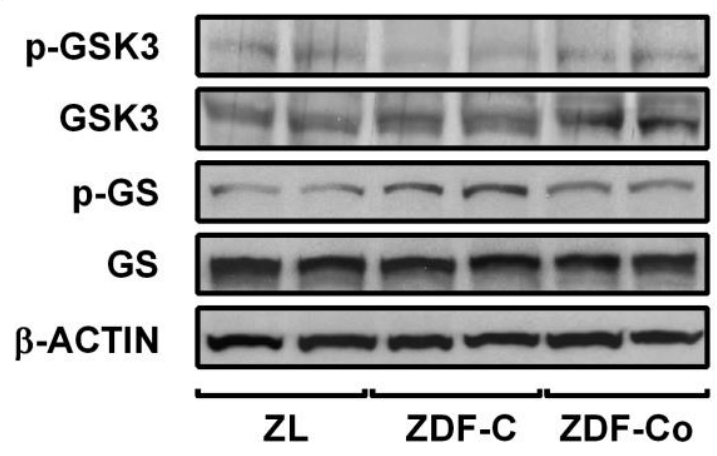

C)

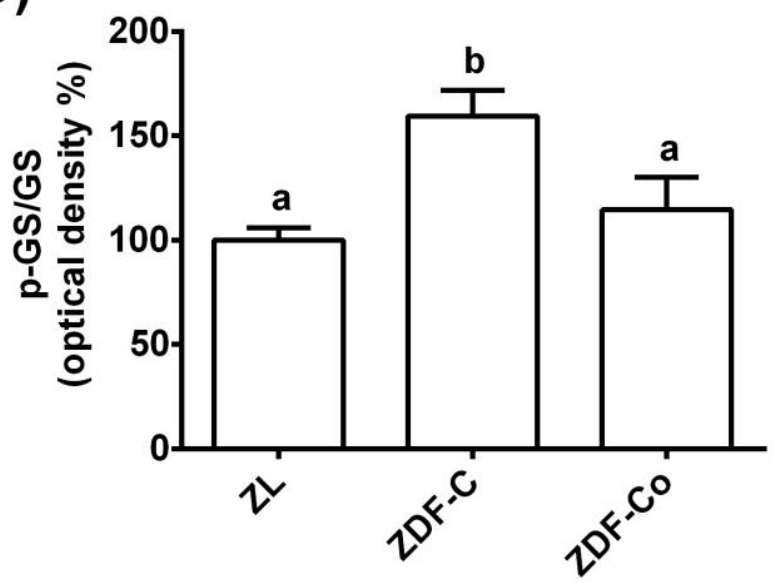

B)

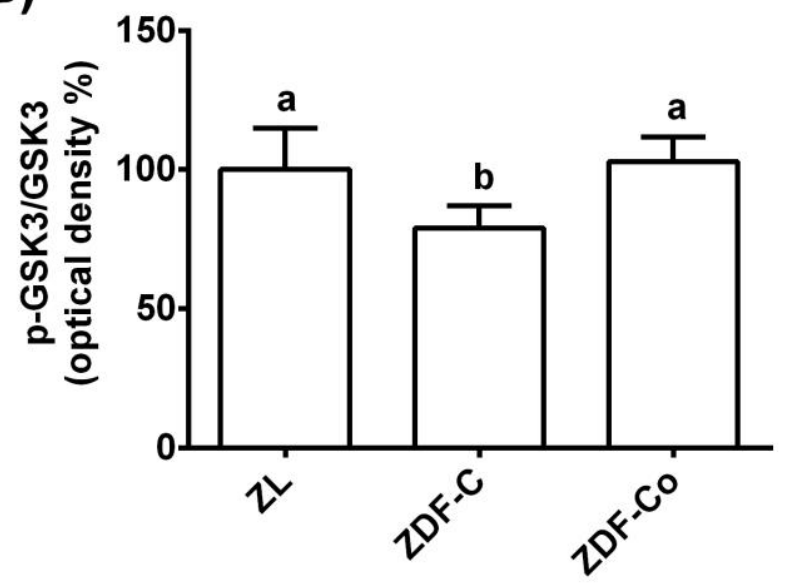

D)

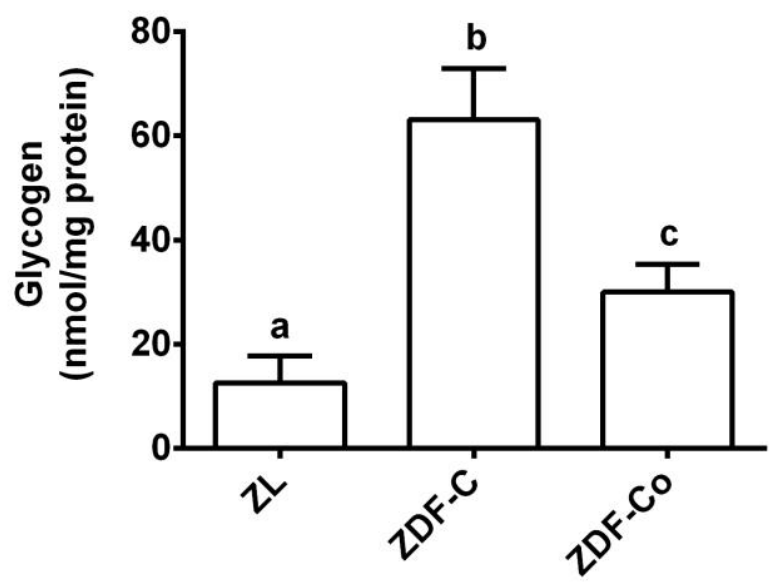


Figure 4

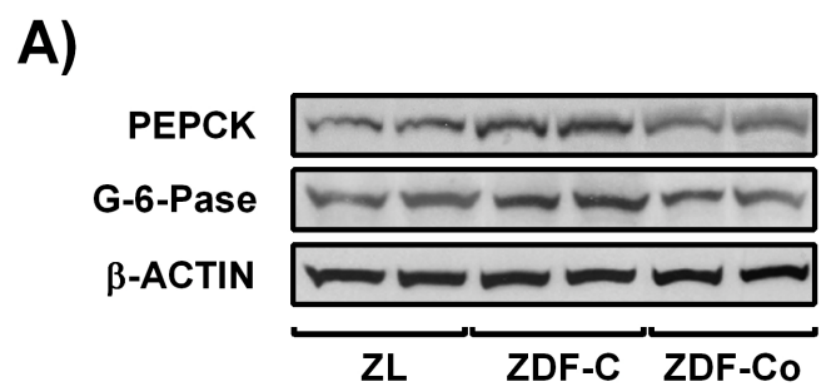

B)

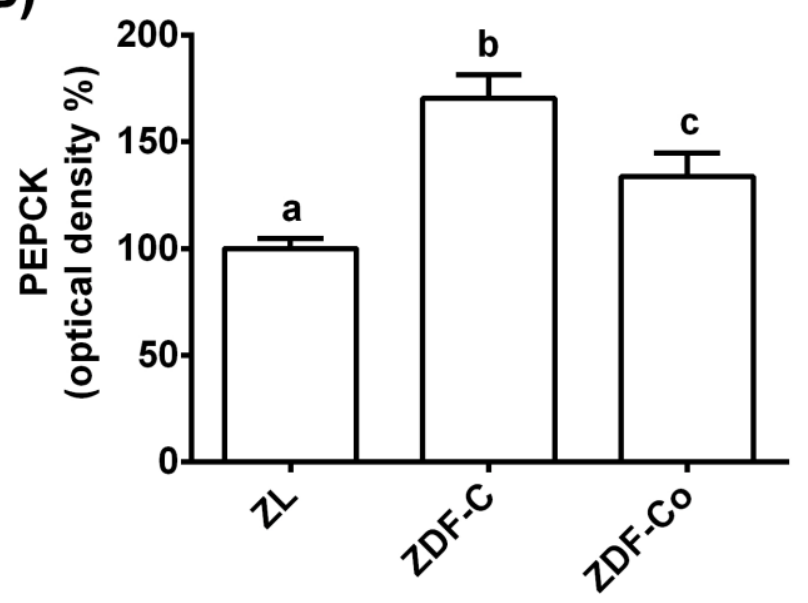

C)

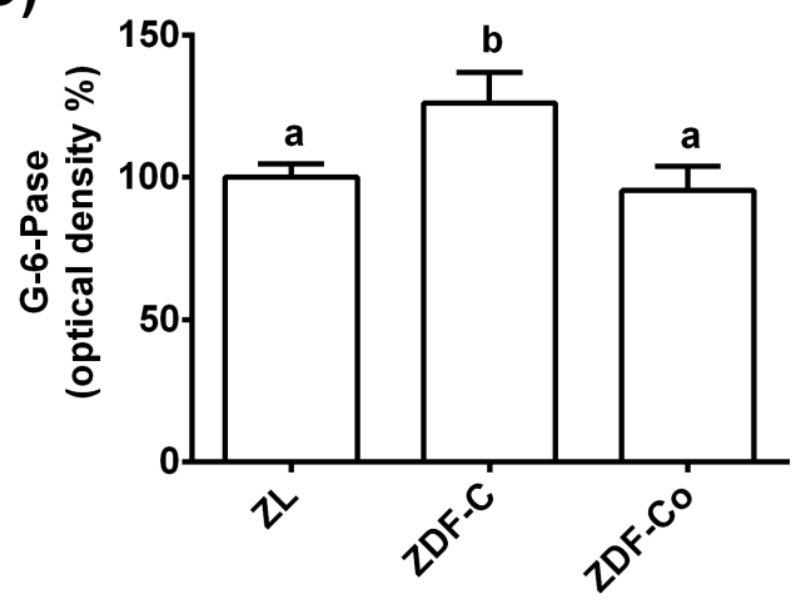


Figure 5

A)

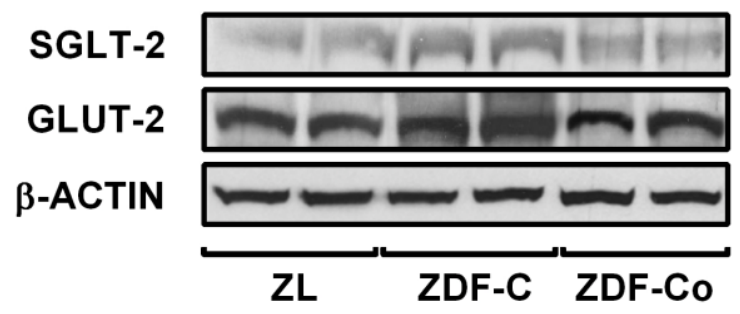

B)

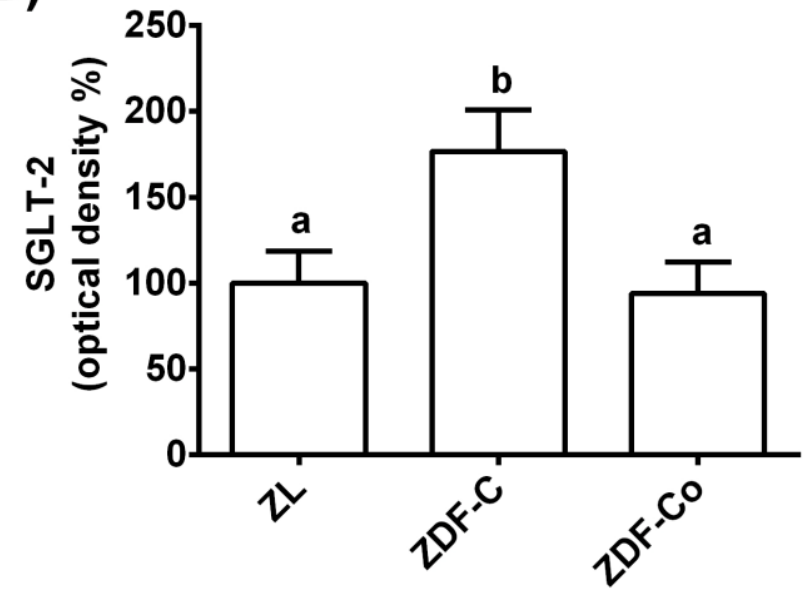

C)

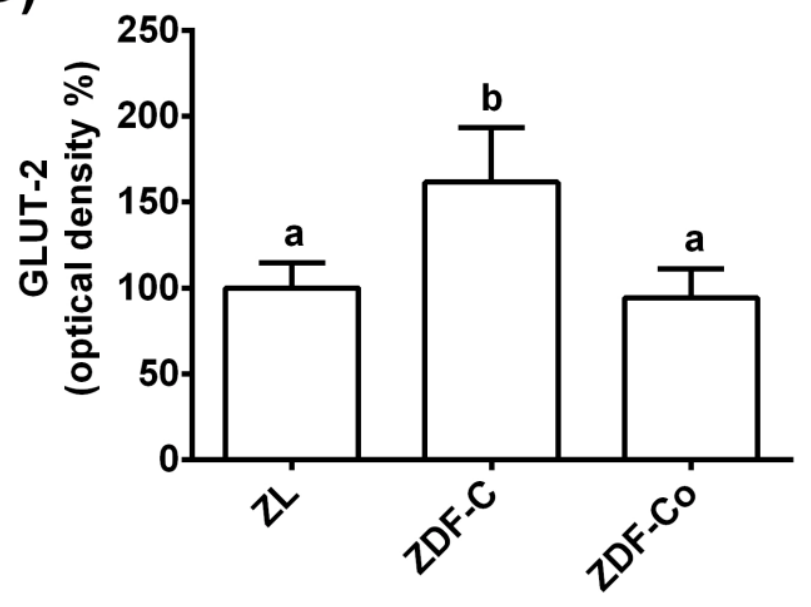

